\title{
Metabolic features of clear-cell renal cell carcinoma: mechanisms and clinical implications
}

\author{
Jehonathan H. Pinthus, MD, PhD; Kaitlyn F. Whelan; Daniel Gallino; Jian-Ping Lu, MD, PhD; \\ Nathan Rothschild, PhD
}

Department of Surgery, Division of Urology, McMaster University, Hamilton, ON

Cite as: Can Urol Assoc J 2011;5(4):274-82; D01:10.5489/cuaj.10196

\begin{abstract}
Central to the malignant behaviour that endows cancer cells with growth advantage is their unique metabolism. Cancer cells can process nutrient molecules differently from normal cells and use it to overcome stress imposed on them by various therapies. This metabolic conversion is controlled by specific genetic mutations that are associated with activation of oncogenes and loss of tumour suppressor proteins. Understanding these processes is important as it can lead to the discovery of biomarkers that can predict the aggressiveness of the disease and its response to therapy, and even more importantly, to the development of novel therapeutics. A classic tumour in this respect is clear-cell renal cell carcinoma (RCC). In this review, we will begin with a brief summary of normal cellular bioenergetic pathways, which will be followed by a description of the characteristic metabolism of glucose and lipids in clear-cell RCC cells and its clinical implications. Data relating to the potential effect of dietary nutrients on RCC will also be reviewed along with potential therapies targeted at interrupting specific metabolic pathways in clear-cell RCC.
\end{abstract}

\section{Résumé}

Le métabolisme unique des cellules cancéreuses est au cœur du comportement malin qui leur confère un avantage sur le plan de la croissance. Les cellules cancéreuses peuvent traiter les molécules de nutriment différemment des cellules normales et utilisent ces molécules pour surmonter le stress imposé par les différents traitements. La conversion métabolique est contrôlée par des mutations génétiques précises associées à l'activation d'oncogènes et à la perte de protéines de suppression tumorale. Il est important de bien saisir ces processus, car leur élucidation peut mener à la découverte de biomarqueurs permettant de prédire l'agressivité de la maladie et la réponse au traitement et, fait encore plus important, elle peut mener à la mise au point de nouveaux médicaments. À cet égard, I'hypernéphrome à cellules claires représente une tumeur classique. Dans cet article, nous commençons par résumer brièvement les voies bioénergétiques cellulaires normales, puis nous poursuivons avec une description du métabolisme caractéristique du glucose et des lipides dans les cellules de I'hypernéphrome à cellules claires et ses répercussions cliniques. Les données associées à l'effet potentiel des nutriments sur l'hypernéphrome à cellules claires seront aussi passées en revue, ainsi que les thérapies ciblées potentielles visant l'interruption de voies métaboliques particulières dans I'hypernéphrome à cellules claires.

\section{Introduction}

The evolution of modern targeted therapy for metastatic renal cell carcinoma (RCC), which revolutionized the natural history of the disease, stemmed from a basic understanding of the molecular basis of the disease. The groundbreaking work of Linehan and colleagues at the National Cancer Institute has defined a unique genetic signature for all RCC subtypes. ${ }^{1}$ In a landmark review, this research group linked these genotypes to corresponding phenotypes that lead to the dysregulation of metabolic pathways involved in oxygen-, iron-, energy-, or nutrient-sensing. ${ }^{2}$ This indicates that RCC is a disease of cell metabolism.

In this review, we will begin with a brief summary of normal cellular bioenergetic pathways, which will be followed by a description of the characteristic metabolism of glucose and lipids in RCC cells. Data relating to the potential effect of dietary nutrients on RCC will be reviewed, as well as potential therapies targeted at interrupting specific metabolic pathways in RCC. An English-based literature review was conducted through PubMED using the following terms: kidney cancer, renal cancer, metabolism, glycolysis, glucose, cholesterol, diet and adipogenesis. Our discussion will focus on clear-cell RCC as this histological subtype of RCC is the most common $(83 \%){ }^{3}$ 


\section{Normal cellular bioenergetics}

\section{Glucose metabolism}

To obtain the energy needed for all cellular activities, cells must transform the energy stored in chemical bonds in nutrients by oxidation of fat, protein or carbohydrate, and convert the energy yielded from this process into the highenergy phosphate bonds of adenosine triphosphate (ATP). The energy stored in ATP is then used for energy-requiring processes. Accordingly, glucose, a principal source of energy, is first converted to pyruvate in the process of glycolysis, which generates two molecules of nicotinamide adenine dinucleotide (NADH). Pyruvate can then be reduced to lactate (anaerobic glycolysis) or converted to acetyl coenzyme A (CoA). Oxidation of fatty acids and many amino acids also converge in the generation of acetyl-CoA, which is oxidized in mitochondria via the tricarboxylic acid (TCA) cycle (Fig. 1). The energy is stored in electron-accepting coenzymes, $\mathrm{NAD}^{+}$and flavin adenine dinucleotide (FAD), which are reduced to $\mathrm{NADH}$ and $\mathrm{FAD}(2 \mathrm{H})$, respectively. $\mathrm{NADH}$ and $\mathrm{FAD}(2 \mathrm{H})$ subsequently transport electrons to oxygen through a series of electron transfer proteins located in the inner mitochondrial membrane (electron transport chain). This process generates an electromechanical potential gradient, which drives the synthesis of ATP by ATP synthase. The end products of the aerobic oxidation of glucose are water and carbon dioxide $\left(\mathrm{CO}_{2}\right)$. When cells have a limited supply of oxygen (hypoxia), they utilize anaerobic glycolysis to generate ATP, and pyruvate is reduced to the end product lactate.

In terms of energy production (determined by the number of ATP molecules generated), glycolysis generates only 2 moles of ATP per mole of glucose whereas complete aerobic oxidation of glucose to $\mathrm{CO}_{2}$ via oxidative phosphorylation generates 36 moles of ATP.

\section{Pentose phosphate pathway}

The pentose phosphate pathway (PPP) generates reducing equivalents in the form of nicotinamide adenine dinucleotide phosphate (NADPH) and ribose-5-phosphate (R5P) (Fig. 2 ). Both of these molecules are used by cancerous cells. $\mathrm{NADPH}$ is used as a reducing agent for reductive detoxification, which is essential for protection against oxidative stress, and R5P is used as a backbone for nucleotide synthesis in the highly proliferating malignant cells. In the oxidative phase of the PPP, the enzyme glucose-6-phosphate dehydrogenase (G6PD) oxidizes G6P and generates NADPH, and subsequently, R5P. In the non-oxidative phase of the PPP, intermediates of glycolysis (glyceraldehyde-3-phosphate [GAP] and fructose-6-phosphate [F6P]) are converted to R5P. However, this pathway is reversible. Thus, if the cell

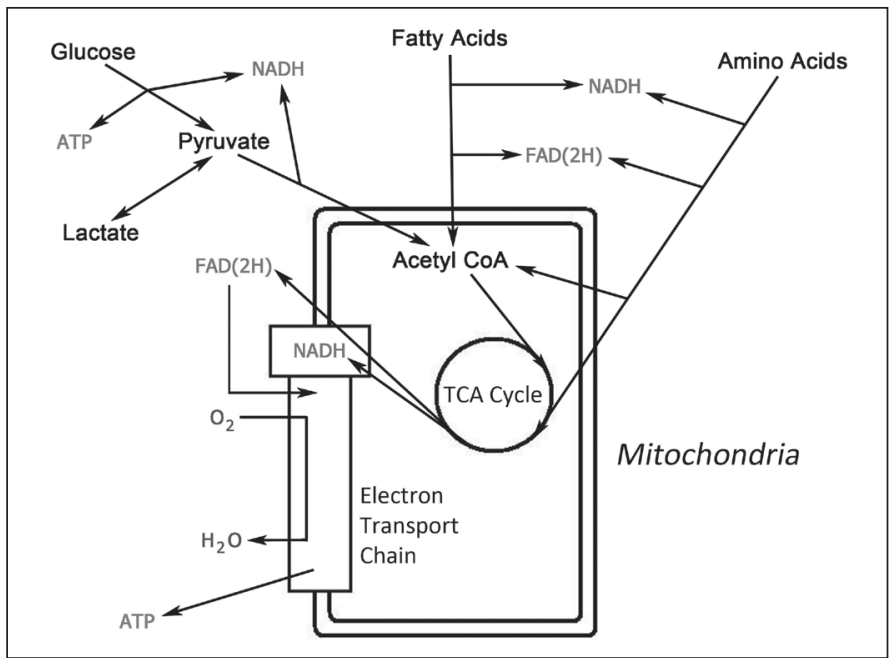

Fig. 1. Cellular bioenergetics.

requires a significant amount of reducing power (NADPH), R5P will be converted back to F6P and GAP by transketolase generating NADPH. This will also occur if the cell has enough R5P for nucleotide synthesis. However, if the cell needs to produce nucleotides, the oxidative pathway of the PPP will be inhibited and glycolytic intermediates will be used to generate R5P in the non-oxidative phase of the PPP.

\section{Lipid and cholesterol synthesis}

Lipogenesis is an important metabolic process in cancer cells. The rapid proliferation of cancer cells creates high demand for membrane "building blocks" (phospholipids and cholesterol). The description of the entire cholesterol synthesis pathway is beyond the scope of this review.

Fatty acids are carboxylic acids with an unbranched aliphatic chain, which is either saturated or unsaturated. Fatty acids are fundamental to energy production and storage, to cellular structure, and as intermediates in molecule biosynthesis. In recent years, it has become apparent that fatty acid synthesis is an active metabolic process in many cancer types. Unlike normal cells that preferentially use dietary (exogenous) lipids for synthesis of endogenous lipids, cancer cells prefer de novo synthesis. ${ }^{4}$ Consequently, cancer cells have elevated expression and activity of key enzymes in this process that can serve as a therapeutic target. ${ }^{4}$ The first step of fatty acid synthesis is the formation of malonyl-CoA by condensation of acetyl-CoA and $\mathrm{CO}_{2}$ catalyzed by acetylCoA carboxylase, which is the major site of the regulation of fatty acid synthesis.

The next steps are catalyzed by cytosolic fatty acid synthase (a multi-enzyme complex), the first of which is condensation of acetyl-acyl carrier protein (ACP) and malonyl-ACP, forming acetoacetyl-ACP. In each round of elongation by the repeated condensation of acetyl-ACP, the $\beta$-keto group is 
Pinthus et al.

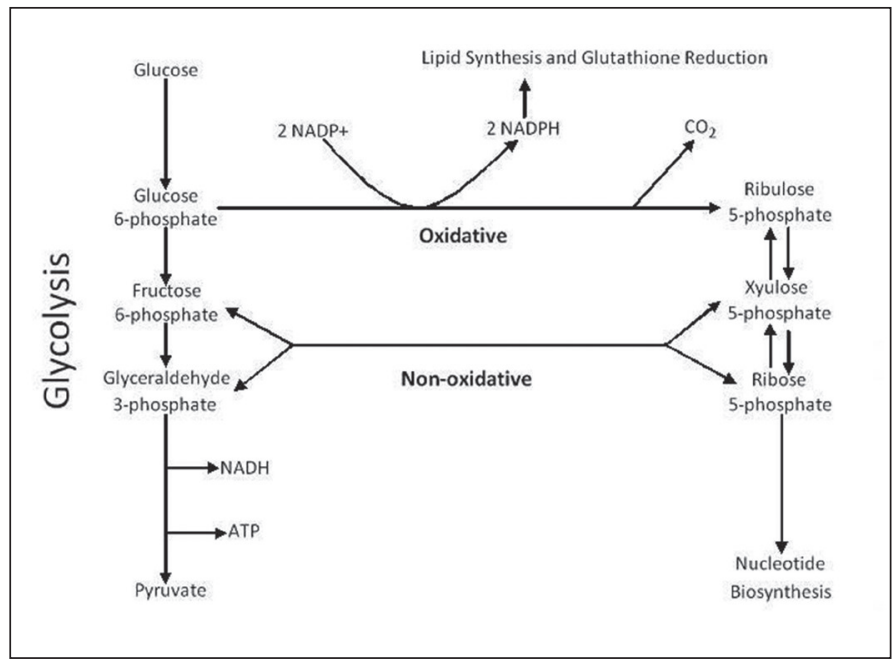

Fig. 2. Pentose phosphate pathway.

reduced to the fully saturated carbon chain by the sequential action of a ketoreductase, dehydratase, and enol reductase. The NADPH that serves as an electron donor in fatty acid synthesis is produced mainly by the PPP.

\section{Metabolic features of clear-cell RCC}

\section{Glucose metabolism}

Unlike normal cells, cancer cells metabolize glucose mostly via glycolysis, even in the presence of sufficient oxygen. ${ }^{5}$ This phenomenon of oncogenic aerobic glycolysis (as opposed to normal anaerobic glycolysis that takes place in the absence of sufficient oxygen) was first described by Warburg and is pathognomonic in clear-cell RCC for several reasons. ${ }^{5}$

Many alterations in the normal cellular hemostasis and metabolism in clear-cell RCC occur in response to pseudohypoxia (the activation of hypoxia response pathways under normal oxygen conditions). Clear-cell RCC typically exhibits this phenomenon because of specific molecular alterations. The major molecular event leading to the development of clear-cell RCC is inactivation or loss of the von HippelLindau (VHL) tumour suppression gene. ${ }^{6,7}$ Briefly, the VHL protein is part of the E3-ubiquitin ligase complex that binds to the hypoxia-inducible factor subunit $\alpha$ (HIF- $\alpha$ ) under normoxic conditions and directs it to proteosomal degradation. Thus, in the absence of functional VHL, even under normoxic conditions, HIF- $\alpha$ is stable and translocates to the nucleus to form a dimer with HIF- $\beta$ to generate HIF-1, which binds to the hypoxia response element (HRE) motif of target genes to activate or suppress their transcription. Increased levels of HIF-1 resulting in upregulation of its target proteins equip clear-cell RCC cells with a specific set of enzymatic machinery that favours aerobic glycolysis over oxidative phosphory- lation. ${ }^{8}$ One of the genes controlled by HIF-1 (because it contains an HRE site) is glucose transporter 1 (Glut1), which encodes a membrane transporter of glucose. In clear-cell RCC, upregulation of Glut1 expression results in an influx of glucose to the cancer cells. ${ }^{9}$ Additionally, the transcription of several other key enzymes in the glycolytic pathway is stimulated by HIF. A classic example is the effect of HIF on lactate production from pyruvate, which is integral to the process of enhanced glycolysis. This process occurs through the enzymatic activity of lactate dehydrogenase, which converts pyruvate to lactate. Lactate is then exported out of the cell by its carrier, monocarboxylate transporter 4 (MCT4). Both $\mathrm{LDH}$ and MCT4 are regulated by HIF. ${ }^{10,11}$ Lactate production promotes a malignant phenotype through induction of several oncogenes and the creation of an acidic environment that protects cancer cells from the immune system. ${ }^{12}$ In addition, lactate has been shown to stimulate HIF- $1 \alpha$ accumulation, thus augmenting HIF-1-regulated glycolysis in RCC. Interestingly, inhibition of LDH-A, a major isoform of $\mathrm{LDH}$ and a known HIF target which is overexpressed in RCC, has been recently shown to suppress the tumouregenesis of cells that underexpress fumarate hydratase $(\mathrm{FH})$ both in vitro and in vivo. ${ }^{13}$ Inactivation of the $\mathrm{FH}$ gene, a key enzyme in the TCA cycle which catalyzes the conversion of fumarate to malate, results in the accumulation of HIF. ${ }^{14}$ Although the inhibitory effects of $\mathrm{LDH}-\mathrm{A}$ inhibition were shown for the smaller subset of type 2 papillary RCC which have a genetic defect in the $\mathrm{FH}$ gene (patients with hereditary leiomyomatosis and renal cell cancer- HLRCC), they are probably applicable to the more common type of clearcell RCC, in which enhanced LDH activity and glycolysis occurs also largely due to HIF activity. Importantly, aside from enhanced glycolysis, over-activity of HIF also inhibits oxidative phosphorylation. Pyruvate dehydrogenase kinase 1 (PDK-1) phosphorylates the enzyme pyruvate dehydrogenase to inhibit its activity. Pyruvate dehydrogenase catalyzes the conversion of pyruvate to acetyl-CoA, the entry product for oxidative phosphorylation (Fig. 1). Oxidative phosphorylation is therefore inhibited in clear-cell RCC because PDK-1 is a HIF target. ${ }^{15}$ Accordingly, exciting experiments by Hervouet and colleagues demonstrate in vitro that introduction of VHL into VHL-/- clear-cell RCC cells restores oxidative phosphorylation. ${ }^{16}$

Another common molecular event in clear-cell RCC that leads to increased glycolysis and reduced oxidative phosphorylation is the enhanced signaling pathways governed by Akt. The PI3K/Akt signaling pathway is constitutively active in kidney cancer. ${ }^{17}$ Akt hyperactivation is closely associated with cellular processes that contribute to oncogenic aerobic glycolysis. Akt-induced glycolysis may be mediated by multiple non-exclusive mechanisms. ${ }^{18}$ Among these mechanisms are induction of expression and membrane translocation of glucose transporters, increased hexokinase expression and 


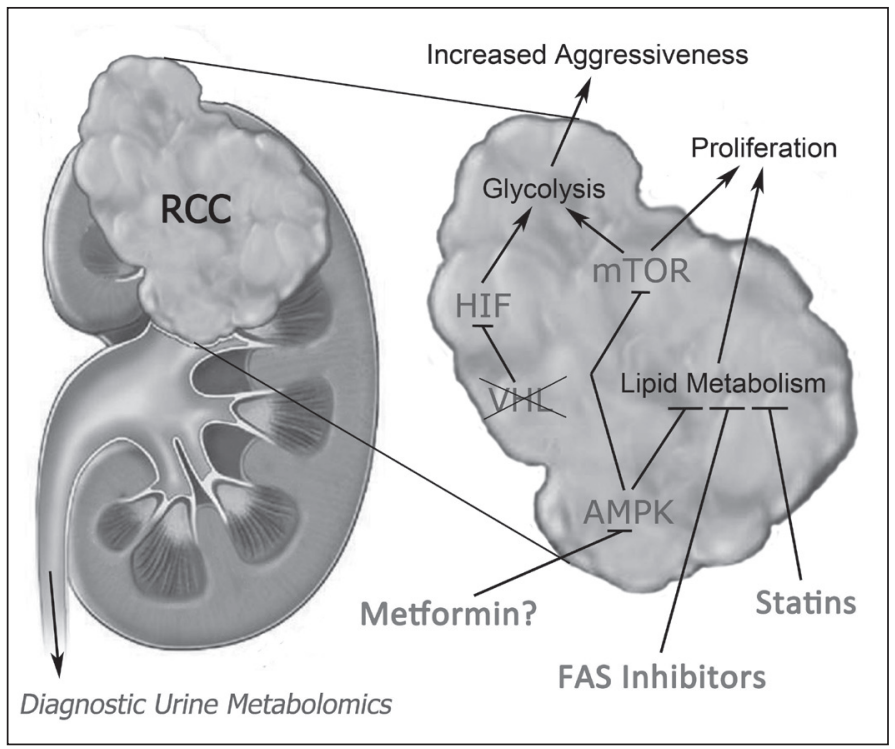

Fig. 3. Kidney cancer as a metabolic disease: potential diagnostics and metabolic modifiers.

activity, and activation of phosphofructokinase 1 (PFK-1). PFK-1 is normally highly regulated as it catalyses the first irreversible reaction in glycolysis. Importantly, Akt hyperactivity can also increase the activity of mammalian target of rapamycin (mTOR), thereby increasing HIF-1-associated glycolytic effects.

In summary, clear-cell RCC cells obtain their energy primarily from aerobic glycolysis rather than mitochondrial oxidative phosphorylation. However, it should be emphasized that although the utilization of anaerobic glycolysis for ATP production (per mole of glucose) is 18 times less efficient than oxidative phosphorylation, clear-cell RCC cells still receive abundant supplies of ATP through aerobic glycolysis to support the rapid and unrestricted proliferation. ${ }^{19}$

\section{Alterations in the pentose phosphate pathway (PPP) and their significance in clear-cell RCC pathogenicity}

Clear-cell RCC cells are endowed with increased activity of the pentose phosphate pathway (PPP). ${ }^{20}$ In addition, clear-cell RCC cells have higher resistance to apoptosis, oxidative stress, and radiation compared with normal cells. These unique characteristics are related to the intensive generation of NADPH by the PPP. The oxidative branch of the PPP converts glucose into ribulose and generates the reducing force $\mathrm{NADPH}$, which is critical in defense against oxidative stress, lipid synthesis, and many other anabolic reactions. Therefore, the PPP is critical in supporting the rapid proliferation of clear-cell RCC. In addition, the PPP generates ribose needed for nucleotide synthesis. RCC has been found to have increased activity of G6PD and transketolase, ${ }^{20}$ the two key enzymes involved in the PPP (Fig.
2). The enhanced expression of transketolase-like 1 (TLKL1) protein - the only transketolase that is overexpressed in cancer-was shown to correlate with a more malignant RCC phenotype in a study by Langbein and colleagues. ${ }^{20}$ These investigators suggested that enhanced transketolase activity induces a shift toward anaerobic glycolysis and promotes growth advantage in hypoxic conditions, and, in particular, may select cells that are resistant to current anti-angiogenesis targeted treatment. ${ }^{21}$

The increased activity of the PPP in clear-cell RCC has a significant role in protecting cancer cells from oxidative stress. Accumulation of reactive oxygen species (ROS) can act against cancer cells by activating apoptosis. NADPH is a cofactor of glutathione reductase, which reduces glutathione disulfide to glutathione. Glutathione then reduces hyperoxide to water, maintaining a low level of oxidative stress in cells. ${ }^{22}$ The production ratio of $\mathrm{NADPH} \mathrm{NADP}^{+}$in the PPP is usually high in rapidly proliferating tumour cells. ${ }^{23,24}$ Consistent with a role for the PPP in NADPH production, an increase in G6PDH activity has been found to be associated with increased protection against oxidative stress in various cell types. ${ }^{25,26}$

\section{Lipid metabolism in clear-cell RCC}

The histological appearance of clear-cell RCC is derived from the cellular accumulations of lipids. ${ }^{27}$ The cellular accumulations of lipids in clear-cell RCC may serve as an anabolic sink for the high rate of NADPH production by the overactive PPP in cancer cells as NADPH is used in lipid synthesis (cholesterol and fatty acids). Nevertheless, in many tumour cells, altered lipid metabolism is reported, and there is evidence to support lipogenesis being part of the malignant process of RCC, ${ }^{28}$ as it is in many other cancers. Fatty acid synthase (FAS) is a key enzyme involved in the de novo biosynthesis of fatty acids, and over-expression of FAS is common in many cancers. Accumulating evidence suggests that it is a metabolic oncogene with an important role in tumour growth and survival. ${ }^{29}$ Horiguchi and colleagues reported that FAS over-expression is an indicator of tumour aggressiveness and poor prognosis in RCC. ${ }^{30}$ Although only $15 \%$ of the tumours stained positively for FAS expression in their immunohistochemical analysis, positive staining was significantly associated with advanced pathological T stage regional lymph node metastasis, distant metastasis, higher histological grade, microvascular invasion and shorter cancer-specific survival compared with negative FAS expression. ${ }^{30}$ The same group demonstrated in preclinical in vitro and in vivo models of RCC that the pharmacological FAS inhibitor C75 can significantly inhibit cellular viability and growth by arresting the cell cycle at the G2/M phase and by induction of apoptosis. ${ }^{31}$ They also found that systemic administration of C75 can inhibit RCC xenografts in mice. ${ }^{31}$ 
Pinthus et al.

A number of novel compounds that specifically inhibit FAS are under development. ${ }^{32}$

It should be noted that FAS is the only enzyme that is able to synthesize fatty acids de novo. ${ }^{32}$ Other enzymes in the pathway such as acetyl-CoA carboxylase (ACC) which catalyzes the carboxylation of acetyl-CoA to Malonyl-CoA, can serve as a target. Indeed drugs (like metformin) that activate 5'-AMP-activated protein kinase (AMPK) induce inhibition of ACC. AMPK phosphorylates and inhibits ACC1 and therefore suppresses fatty acid synthesis. ${ }^{33}$

Clear-cell RCC cells contain an excess of total cholesterol and, more specifically, esterified cholesterol (8-fold and 35 -fold more than is found in normal kidneys, respectively). ${ }^{34}$ Although free cholesterol accumulation is toxic to cells, RCC cells are protected against it due to an increase in activity of acetyl-CoA cholesterol acetyl transferase (ACAT). ${ }^{35}$ This enzyme catalyzes the formation of cholesterol esters. Accordingly, it has been shown that specific inhibition of ACAT can inhibit the survival of glioma tumour cells, ${ }^{36}$ but there are currently no similar reports for RCC. Drabkin and Gemmill suggested that the possible link between the relative deficiency of serum adiponectin and aggressive features of RCC ${ }^{37}$ may be explained by the lack of negative regulation that is normally exerted by adiponectin on ACAT expression. ${ }^{38}$

\section{Clinical implications of RCC metabolism}

The unique alterations manifested in clear-cell RCC can be harnessed for specific diagnostic, prognostic and potential therapeutic purposes.

\section{Diagnostic implications}

From a diagnostic standpoint, the application of functional imaging for RCC diagnosis and staging is a classic example. Functional imaging exploits unique metabolic processes within tumour cells, such as increased glycolysis, to delineate tumours. The information provided is not just anatomical but also functional and, as such, can improve the differentiation of RCC from benign primary lesions, reactive changes from therapy, such as lymphadenopathy or scaring, and systemic potentially unrelated processes (such as incidental lung nodules). More importantly, in the current era of successful targeted therapy for mRCC, which results in long-term disease stabilization, functional imaging data are of importance in determining the response to therapy and prognosis. ${ }^{39}$ Positron emission tomography (PET), particularly when combined with computerized tomography (CT), is a promising modality that can provide such information. Fluorodeoxyglucose (FDG) is most commonly used in imaging; it assembles the radiotracer fluorine-18 with an analog of D-glucose. The increased uptake and accumulation of
FDG within certain types of tumour cells, particularly RCC, is due to an increased number of glucose transporter proteins and increased glycolysis. ${ }^{9}$ In the normal glycolytic pathway, FDG behaves similarly to D-glucose. Thus, following its transmembrane transportation, it is phosphorylated by hexokinase. The expression of hexokinase II in clear-cell RCC is elevated. ${ }^{40}$ Once FDG is phosphorylated, structural changes made by a hexose-phosphate bond prevent FDG from being catabolized or transported back into the extracellular space in substantial amounts. ${ }^{41}$ This "metabolic trapping" of FDG highlights the presence and location of RCC. Unfortunately, because FDG is primarily secreted by the kidneys, its role in the diagnosis of primary RCC is limited; however, the sensitivity for primary RCC detection can be improved by hybrid imaging with PET/CT scanners. ${ }^{42}$ For example, in a small study FDG-PET was shown to be valuable in the differential diagnosis of complex renal cysts. ${ }^{43}$ The greatest advantage of using FDG-PET is in the detection of metastatic disease. ${ }^{39,44}$ It is particularly attractive for the differential diagnosis between reactive lymphadenopathy and lymph node metastasis, as well as differentiating between tumour thrombus and benign clot, and between fibrosis of the renal fossa and recurrence post nephrectomy. It is difficult to diagnose these clinical scenarios using only anatomical imaging modalities (CT or magnetic resonance imaging scans). Early reports suggest that FDG-PET/CT findings correlate with the response of RCC to targeted therapy in mRCC. ${ }^{45}$ In general, FDG-PET scans allows us to predict the response to therapy early in the course of treatment, as well as to determine the viability of residual masses after completion of treatment in various cancers. ${ }^{46}$ These data are currently limited for mRCC patients, but the technique appears to be promising. ${ }^{45,47,48}$

FDG-PET scans reflect changes in the metabolic activity of experimental RCC in preclinical in vivo models that are treated with mTOR inhibitors. ${ }^{49}$ Since HIF is regulated by mTOR and given its major effects on glucose metabolism in clear-cell RCC, FDG-PET scans may provide valuable prognostic data for patients treated with mTOR inhibitors or even help select it for second-line targeted agents, which may be used following treatment with tyrosine kinase inhibitors. ${ }^{50}$

\section{Novel metabolic markers for diagnosis and prognostication}

Although in its infancy, the potential use of metabolomics to capture the unique metabolic signature of RCC is exciting. Using two-dimensional gel electrophoresis and mass spectrometry of conventional RCC tissue in comparison with patient-matched normal kidney cortex tissue, Unwin and colleagues captured a proteomic profile typical of RCC metabolism: ${ }^{51}$ an increase in proteins involved in most steps in the glycolytic pathway and a decrease in the gluconeogenic reactions in combination with a parallel reduction in several mitochondrial enzymes. The potential of metabo- 
lomics to assist in the differentiation between benign and malignant renal lesions, and even more importantly, to provide adjunctive information on the malignant potential of tumours has recently been suggested. Using the "shot gun" approach, which relies on liquid chromatography and tandem mass spectrometry to identify trypsin-digested proteins and subsequent computer analysis to reconstruct proteins contained within the sample, Perroud and colleagues demonstrated that changes in the expression of enzymes in the glycolytic pathway reflect tumour behaviour. ${ }^{52}$ These investigators discovered a proteomic variation as a function of clear-cell RCC tumour grade and, by extension, prognosis. Among the proteins that showed grade-dependent expression, 20 belonged to the glycolysis pathway. This study links the specific metabolic changes that accompany the development and progression of RCC to measurable prognostic data. Moreover, researchers are attempting to develop this approach to become a more clinically applicable ancillary diagnostic method by developing urine metabolomics for metabolic profiling, as well as for biomarker discovery for kidney cancer. ${ }^{53,54}$

\section{Potential role for generic metabolic modifiers}

Metabolic reprogramming of cancer cells can induce antineoplastic effects. Accordingly, in recent years, it has been revealed that the use of generic drugs that interfere with cholesterol and glucose metabolism is associated with a reduction in the risk of certain cancers. Much of the attention has been drawn to statins and metformin.

\section{Statins}

Clear-cell RCC received its name because of the clear microscopic appearance of its cells, which results from the accumulation of cholesterol in the cytoplasm of the malignant cells. ${ }^{34}$ Interestingly, compared with normal cells, clear-cell RCC cells express higher levels of ACAT, which esterifies cholesterol. Since free cholesterol (unlike cholesterol esters) is toxic to cells, the higher expression of ACAT and its documented increased activity ${ }^{35}$ in clear-cell RCC probably protect the cells. ${ }^{34}$ Indeed, in vitro experiments in non-RCC tumour cell lines demonstrated that inhibition of ACAT results in tumour suppression. ${ }^{55}$ Collectively, it appears that the accumulation of cholesterol esters in clear-cell RCC is associated with its malignant phenotype. The question as to whether the use of cholesterol synthesis inhibitors may be therapeutic in RCC is raised. This question is particularly relevant as the expression of the low-density lipoprotein receptor that is responsible for the intracellular uptake of cholesterol is regulated by $\mathrm{mTOR}^{56}{ }^{56}$ an active signaling pathway in RCC. ${ }^{57}$ Statins are synthetic agents that inhibit the rate-limiting enzyme of the mevalonate pathway, 3-hydroxy-3-methylglutaryl-CoA reductase, thereby inhibiting cholesterol biosynthesis. ${ }^{58}$ Recently, it has been suggested that statins may have activity against RCC. Khurana and colleagues examined the association between the risk of RCC and use of statins in a retrospective nested case-control study of 483733 veterans, of whom 164441 (34\%) were taking statins prior to the diagnosis of RCC and $1446(0.3 \%)$ had a primary diagnosis of RCC. ${ }^{59}$ The investigators found that statin use was significantly associated with a risk reduction of RCC of $48 \%$ (adjusted odds ratio $0.52,95 \%$ confidence interval $0.45-0.60$ ), irrespective of the presence of obesity. Few studies have examined the potential advantage of statins in preclinical models of RCC. Horiguchi and colleagues demonstrated that oral fluvastatin, administered at the recommended doses for hypercholesterolemia, markedly suppressed metastasis in a mouse model of RCC. ${ }^{60}$ Their in vitro data linked fluvastatin effects to the induction of cell arrest and apoptosis as well as to an inhibitory effect on angiogenesis and invasion. ${ }^{60}$ In another study, Woodard and colleagues demonstrated that statins suppress activation of the mTOR pathway in RCC cells via inhibition of Akt activation. ${ }^{61}$ Although, to our knowledge, there are no data yet to show whether statins can reduce cholesterol accumulation in RCC and whether that by itself has a tumour-suppressive effect, the data suggest a rationale to prospectively examine the role of statins in chemoprevention of primary RCC and against the potential recurrence of the disease following surgical removal of the primary tumour in an adjuvant setting.

\section{Metformin}

Metformin is a widely used oral hypoglycemic agent in type II diabetes. The anti-diabetic effects of this drug are attributed mainly to reduced hepatic glucose production and the induction of increased insulin sensitivity via the activation of AMPK, which is a key negative regulator of mTOR. The AMPK/mTOR axis is the main energy-sensor pathway of the cell and its activation results in mTOR inhibition and, consequently, the shutdown of energy-consuming processes and the activation of energy-producing processes. ${ }^{62}$ As such, metformin can inhibit the metabolic effects induced by constitutive activation of mTOR in $\mathrm{RCC}^{57}$ and its proproliferative effects. Likewise, recent data from epidemiological, animal and in vitro studies suggest that metformin has an anti-proliferative effect in cancer. ${ }^{63}$ Also as discussed previously, activation of AMPK by metformin also inhibits lipogenesis as AMPK inhibits ACC - the rate limiting enzyme in the process of fatty acid synthesis (Fig. 3). Evans and colleagues found that the use of metformin in patients with diabetes was associated with a reduced risk of all cancers; ${ }^{64}$ Bowker and colleagues demonstrated a reduced cancer- 
Pinthus et al.

related mortality rate in patients with diabetes who were receiving metformin compared with diabetic patients who were taking insulin or sulfonylurea. ${ }^{65}$ Most observations have been made for breast and prostate cancer patients. ${ }^{63}$ Only one study to date examined the in vitro effects of metformin on the tumoureginicity of 786-0 RCC cells. ${ }^{66}$ This study demonstrated an inhibitory effect of metformin on the cellular proliferation and anchorage-independent growth characteristics. These effects were synergized by fluvastatin. ${ }^{66}$

Although, in principle, metformin can reprogram the metabolism of cancer cells toward anti-neoplastic effects, the effect of metformin varies between different cancer cell types. ${ }^{63}$ Thus, pre-clinical in vitro and animal testing of the effect of metformin on several RCC models is needed.

\section{Effect of diet on RCC}

Given the roles of glucose and lipid metabolism, as well as oxidative stress in RCC, the potential effects of diet on the development of disease come into question. The exact association between diet and the risk of RCC is unclear. Several case-control and cohort studies have yielded conflicting observations. Maclure and Willett reported that in a case-control study of diet and the risk of RCC involving 203 incident cases, 207 prevalent cases and 605 controls, incident cases consumed more meats and fewer vegetables than controls. ${ }^{67}$ In addition, consumption of saturated and animal fat was significantly associated with the incidence of RCC. Interestingly, cholesterol intake was not associated with any particular risk. Contrary to these results, the authors of a larger, (1185 incident cases, 1526 controls) multicentre, population-based case-control study conducted in Australia, Denmark, Sweden, and the United States reported no associations between the intake of total fat, saturated fat, monounsaturated fat and cholesterol with RCC. ${ }^{68}$ However, a significant positive association was observed between total energy intake and RCC. This is an interesting observation as recent studies have linked excess caloric intake with cancer development, ${ }^{69,70}$ but a specific association with RCC has not been reported. Finally, Lee and colleagues conducted a pooled analysis of 13 prospective studies that examined the associations between meat, fat, and protein intake and the risk of RCC. ${ }^{71}$ Their pooled cohort included 530469 females and 244483 males who completed a validated dietary questionnaire at study entry and were followed for up to 7 to 20 years. A total of 1478 incident cases of RCC were identified. After adjusting for body mass index, fruit and vegetable intake and alcohol intake, they found no association between fat and protein intake and the risk of RCC.

The association between dietary antioxidant intake and RCC is conflicting. Lee and colleagues prospectively examined whether the intake of fruits, vegetables, carotenoids, and vitamins $\mathrm{A}, \mathrm{C}$, and $\mathrm{E}$ was associated with a lower risk of RCC in two large cohorts: the NHS (Nurses' Health Study; 121
700 female participants, 132 cases followed up for 20 years) and the HPFS (Health Professionals Follow-up Study; 51529 male participants, 116 cases followed up for 16 years). ${ }^{72}$ They found that the consumption of fruits and vegetables is associated with a lower risk of RCC in men but not in women. The authors were unable to separate the effect of carotenoids from the effects of fruits and vegetables due to high correlations between carotenoids and fruits and vegetables. Nevertheless, a case-control study based on mailed dietary questionnaires recently showed that a diet rich in $\beta$-carotene and lutein/ zeaxanthin may play a role in RCC prevention. ${ }^{73}$ Conversely, no association between dietary intake of fruits, vegetables, carotenoids, flavonoids, vitamin $\mathrm{E}$, and vitamin $\mathrm{C}$ and the risk of developing RCC was found in the ATBC (AlphaTocopherol, Beta-Carotene Cancer Prevention) study (27 062 male Finnish smokers, 255 cases followed up for 19 years). ${ }^{74}$ Similarly, in the NLCS (Netherlands Cohort Study) on diet and cancer, van Dijk and colleagues reported no association between carotenoid, vitamin or supplemental vitamin intake and the risk of RCC. ${ }^{75}$ Thus, although metabolic alterations in oxidative stress pathways and fat metabolism are definitely part of the RCC genotype and phenotype, the available data do not support a role for dietary manipulations in altering the risk of developing RCC.

\section{Conclusions}

Clear-cell RCC is a glycolytic and lipogenic tumour. Hence, improved diagnostic techniques, novel biomarkers and potential experimental therapeutics for clear-cell RCC should be developed based on these metabolic alterations that distinguish RCC from other tissues, and, specifically, from the normal kidney.

Competing interests: None declared.

Acknowledgement: The authors would like to thank Paladin Canada for their support given to Kaitlyn Whelan through an Educational Grant.

This paper has been peer-reviewed.

\section{References}

1. Rosner I, Bratslavsky G, Pinto PA, et al. The clinical implications of the genetics of renal cell carcinoma. Urol Oncol 2009;27:131-6.

2. Linehan WM, Srinivasan R, Schmidt LS. The genetic basis of kidney cancer: a metabolic disease. Nat Rev Urol 2010;7:277-85.

3. Cheville IC, Lohse CM, Zincke H, et al. Comparisons of outcome and prognostic features among histologic subtypes of renal cell carcinoma. Am I Surg Pathol 2003;27:612-24.

4. Mashima T, Seimiya H, Tsurvo T. De novo fatty-acid synthesis and related pathways as molecular targets for cancer therapy. Br J Cancer 2009;100:1369-72.

5. Warburg 0. On respiratory impairment in cancer cells. Science 1956;124:269-70. 
6. Gleadle JM. Review article: How cells sense oxygen: lessons from and for the kidney. Nephrology (Carlton) 2009; 14:86-93.

7. Baldewiins MM, van Vlodrop IJ, Vermeulen PB, et al. VHL and HIF signalling in renal cell carcinogenesis. J Pathol 2010;221:125-38

8. Godinot $C$, de Laplanche $E$, Hervouet $E$, et al. Actuality of Warburg's views in our understanding of renal cancer metabolism. J Bioenerg Biomembr 2007:39:235-41.

9. Ozcan A, Shen SS, Zhai QJ, et al. Expression of GLUT1 in primary renal tumours: morphologic and biologic implications. Am I Clin Pathol 2007;128:245-54.

10. Firth JD, Ebert BL, Ratcliffe PJ. Hypoxic regulation of lactate dehydrogenase A. Interaction between hypoxia-inducible factor 1 and cAMP response elements. J Biol Chem 1995;270:21021-7.

11. Ullah MS, Davies AJ, Halestrap AP. The plasma membrane lactate transporter MCT4, but not MCT1, is up-regulated by hypoxia through a HIF-lalpha-dependent mechanism. J Biol Chem 2006;281:9030-7.

12. Gatenby RA, Gillies RJ. Why do cancers have high aerobic glycolysis? Nat Rev Cancer 2004;4:891-9.

13. Xie H, Valera VA, Merino MJ, et al. LDH-A inhibition, a therapeutic strategy for treatment of hereditary leiomyomatosis and renal cell cancer. Mol Cancer Ther 2009;8:626-35.

14. Isaacs JS, Jung YJ, Mole DR, et al. HIF overexpression correlates with biallelic loss of fumarate hydratase in renal cancer: novel role of fumarate in regulation of HIF stability. Cancer Cell 2005;8:143-53.

15. Papandreou I, Cairns RA, Fontana L, et al. HFF-1 mediates adaptation to hypoxia by actively downregulating mitochondrial oxygen consumption. Cell Metab 2006;3:187-97.

16. Hervouet E, Demont J, Pecina P, et al. A new role for the von Hippel-Lindau tumour suppressor protein: stimulation of mitochondrial oxidative phosphorylation complex biogenesis. Carcinogenesis 2005;26:531-9.

17. Park JY, Lin PY, Weiss RH. Targeting the PI3K-Akt pathway in kidney cancer. Expert Rev Anticancer Ther 2007:7:863-70.

18. Robey RB, Hay N. Is Akt the "Warburg kinase"?-Aktenergy metabolism interactions and oncogenesis. Semin Cancer Biol 2009;19:25-31.

19. Bartrons R, Caro J. Hypoxia, glucose metabolism and the Warburg's effect. J Bioenerg Biomembr 2007;39:223-9.

20. Langbein S, Frederiks WM, zur Hausen A, et al. Metastasis is promoted by a bioenergetic switch: new targets for progressive renal cell cancer. Int I Cancer 2008;122:2422-8.

21. Sharma SG, Nanda S, Longo S. Anti-angiogenic therapy in renal cell carcinoma. Recent Pat Anticancer Drug Discov 2010;5:77-83.

22. Spitz MR, Duphorne $C M$, Detry MA, et al. Dietary intake of isothiocyanates: evidence of a joint effect with glutathione S-transferase polymorphisms in lung cancer risk. Cancer Epidemiol Biomarkers Prev 2000;9:1017-20.

23. Klein A, Chan AW, Caplan BU, et al. NADP+ reduction by human lymphocytes. Clin Exp Immunol 1990;82:170-3

24. Bernstein $\mathrm{H}$, Holubec $\mathrm{H}$, Warneke JA, et al. Patchy field defects of apoptosis resistance and dedifferentiation in flat mucosa of colon resections from colon cancer patients. Ann Surg Oncol 2002; 9:505-17.

25. Bolanos JP, Delgado-Esteban M, Herrero-Mendez A, et al. Regulation of glycolysis and pentose-phosphate pathway by nitric oxide: impact on neuronal survival. Biochim Biophys Acta 2008; 1777:789-93

26. Scott MD, Zuo L, Lubin BH, et al. NADPH, not glutathione, status modulates oxidant sensitivity in normal and glucose-6-phosphate dehydrogenase-deficient erythrocytes. Blood 1991;77:2059-64.

27. Rezende RB, Drachenberg CB, Kumar D, et al. Differential diagnosis between monomorphic clear cell adenocarcinoma of salivary glands and renal (clear) cell carcinoma. Am J Surg Pathol 1999;23:1532-8.

28. Tun HW, Marlow LA, von Roemeling $C A$, et al. Pathway signature and cellular differentiation in clear cell renal cell carcinoma. PLoS One 2010;5:e10696.

29. Flavin R, Peluso $S$, Nguyen PL, et al. Fatty acid synthase as a potential therapeutic target in cancer. Future Oncol 2010;6:551-62.

30. Horiguchi A, Asano T, Ito K, et al. Fatty acid synthase over expression is an indicator of tumour aggressiveness and poor prognosis in renal cell carcinoma. J Urol 2008;180:1137-40.

31. Horiguchi A, Asano T, Ito K, et al. Pharmacological inhibitor of fatty acid synthase suppresses growth and invasiveness of renal cancer cells. J Urol 2008; 180:729-36.

32. Kridel SJ, Lowther WT, Pemble CW 4th. Fatty acid synthase inhibitors: new directions for oncology. Expert Opin Investig Drugs 2007;16:1817-29.

33. Steinberg GR, Kemp BE. AMPK in Health and Disease. Physiol Rev 2009;89:1025-78.

34. Gebhard RL, Clayman RV, Prigge WF, et al. Abnormal cholesterol metabolism in renal clear cell carcinoma. J Lipid Res 1987;28:1177-84.

35. Matsumoto K, Fuijwara Y, Nagai R, et al. Expression of two isozymes of acy-coenzyme A: cholesterol acyltransferase-1 and - 2 in clear cell type renal cell carcinoma. Int J Urol 2008;15:166-70.

36. Bemlih S, Poirier MD, El Andaloussi A. Acyl-coenzyme A: cholesterol acyltransferase inhibitor Avasimibe affect survival and proliferation of glioma tumour cell lines. Cancer Biol Ther 2010;9:1025-32.

37. Pinthus JH, Kleinmann N, Tisdale B, et al. Lower plasma adiponectin levels are associated with larger tumour size and metastasis in clear-cell carcinoma of the kidney. Eur Urol 2008;54:866-73.

38. Drabkin HA, Gemmill RM. Obesity, cholesterol, and clear-cell renal cell carcinoma (RCC). Adv Cancer
Res 2010;107:39-56.

39. Lawrentschuk N, Davis ID, Bolton DM, et al. Functional imaging of renal cell carcinoma. Nat Rev Urol 2010;7:258-66.

40. Adams V, Kempf $\mathrm{W}$, Hassam $\mathrm{S}$, et al. Determination of hexokinase isoenzyme I and II composition by RT-PCR: increased hexokinase isoenzyme Il in human renal cell carcinoma. Biochem Mol Med 1995;54:53-8.

41. Kostakoglu L, Agress H Jr, Goldsmith SJ. Clinical role of FDG PET in evaluation of cancer patients. Radiographics 2003;23:315-40; quiz 533.

42. Perini R, Pryma D, Divgi C. Molecular imaging of renal cell carcinoma. Urol Clin North Am 2008;35(4):60511 ; vi.

43. Goldberg MA, Mayo-Smith WW, Papanicolaou N, et al. FDG PET characterization of renal masses: preliminary experience. Clin Radiol 1997;52:510-5.

44. Ramdave S, Thomas GW, Berlangieri SU, et al. Clinical role of F-18 fluorodeoxyglucose positron emission tomography for detection and management of renal cell carcinoma. J Urol 2001;166:825-30.

45. Vercellino L, Bousquet G, Baillet $G$, et al. 18F-FDG PET/CT imaging for an early assessment of response to sunitinib in metastatic renal carcinoma: preliminary study. Cancer Biother Radiopharm 2009;24:137-44.

46. Avril NE, Weber WA. Monitoring response to treatment in patients utilizing PET. Radiol Clin North Am 2005; $43: 189-204$.

47. Jennens RR, Rosenthal MA, Lindeman GJ, et al. Complete radiological and metabolic response of metastatic renal cell carcinoma to SU5416 (semaxanib) in a patient with probable von Hippel-Lindau syndrome. Urol Oncol 2004:22:193-6.

48. Lyrdal D, Boiisen M, Suurkula $M$, et al. Evaluation of sorafenib treatment in metastatic renal cell carcinoma with 2-fluoro-2-deoxyglucose positron emission tomography and computed tomography. Nucl Med Commun 2009;30:519-24.

49. Thomas GV, Tran C, Mellinghoff IK, et al. Hypoxia-inducible factor determines sensitivity to inhibitors of mTOR in kidney cancer. Nat Med 2006;12:122-7.

50. Motzer RJ, Escudier B, Oudard S, et al. Efficacy of everolimus in advanced renal cell carcinoma: a doubleblind, randomised, placebo-controlled phase III trial. Lancet 2008;372:449-56.

51. Unwin RD, Craven RA, Harnden P, et al. Proteomic changes in renal cancer and co-ordinate demonstration of both the glycolytic and mitochondrial aspects of the Warburg effect. Proteomics 2003;3:1620-32.

52. Perroud B, Lee J, Valkova N, et al. Pathway analysis of kidney cancer using proteomics and metabolic profiling. Mol Cancer 2006:5:64.

53. Kind T, Tolstikov V, Fiehn 0 , et al. A comprehensive urinary metabolomic approach for identifying kidney cancerr. Anal Biochem 2007:363:185-95.

54. Kim K, Aronov P, Zakharkin SO, et al. Urine metabolomics analysis for kidney cancer detection and biomarker discovery. Mol Cell Proteomics 2009:8:558-70.

55. Paillasse MR, de Medina P, Amouroux $G$, et al. Signaling through cholesterol esterification: a new pathway for the cholecystokinin 2 receptor involved in cell growth and invasion. J Lipid Res 2009:50:2203-11.

56. Sharpe L, Brown AJ. Rapamycin down-regulates LDL-receptor expression independently of SREBP-2. Biochem Biophys Res Commun 2008;373:670-4.

57. Robb VA, Karbowniczek M, Klein-Szanto AJ, et al. Activation of the mTOR signaling pathway in renal clear cell carcinoma. J Urol 2007;177:346-52.

58. Istvan ES, Deisenhofer J. Structural mechanism for statin inhibition of HMG-CoA reductase. Science 2001:292:1160-4.

59. Khurana V, Caldito G, Ankem M. Statins might reduce risk of renal cell carcinoma in humans: case-control study of 500,000 veterans. Urology 2008; 71:118-22

60. Horiguchi A, Sumitomo M, Asakuma J, et al. 3-hydroxy-3-methylglutaryl-coenzyme a reductase inhibitor, fluvastatin, as a novel agent for prophylaxis of renal cancer metastasis. Clin Cancer Res 2004; 10:8648-55.

61. Woodard J, Sassano A, Hay N, et al. Statin-dependent suppression of the Akt/mammalian target of rapamycin signaling cascade and programmed cell death 4 up-regulation in renal cell carcinoma. Clin Cancer Res 2008; 14:4640-9

62. Hardie DG. The AMP-activated protein kinase pathway-new players upstream and downstream. J Cell Sci 2004:117:5479-87.

63. Ben Sahra I, Le Marchand-Brustel Y, Tanti JF, et al. Metformin in cancer therapy: a new perspective for an old antidiabetic drug? Mol Cancer Ther 2010;9:1092-9.

64. Evans JM, Donnelly LA, Emslie-Smith AM, et al. Metformin and reduced risk of cancer in diabetic patients. BMJ 2005:330:1304-5.

65. Bowker SL, Majumdar SR, Veugelers P, et al. Increased cancer-related mortality for patients with type 2 diabetes who use sulfonylureas or insulin. Diabetes Care 2006;29:254-8.

66. Woodard J, Joshi S, Viollet B, et al. AMPK as a therapeutic target in renal cell carcinoma. Cancer Biol Ther 2010;10:1168-77. Epub 2010 Dec 1.

67. Maclure M, Willett W. A case-control study of diet and risk of renal adenocarcinoma. Epidemiology 1990; 1:430-40.

68. Wolk A, Gridley G, Niwa S, et al. International renal cell cancer study. VII. Role of diet. Int J Cancer 1996;65:67-73. 
Pinthus et al.

69. Fair AM, Montgomery K. Energy balance, physical activity, and cancer risk. Methods Mol Biol 2009:472:57-88.

70. Longo VD, Fontana L. Calorie restriction and cancer prevention: metabolic and molecular mechanisms. Trends Pharmacol Sci 2010;31:89-98.

71. Lee JE, Spiegelman D, Hunter DJ, et al. Fat, protein, and meat consumption and renal cell cancer risk: a pooled analysis of 13 prospective studies. J Natl Cancer Inst 2008;100:1695-706.

72. Lee JE, Giovannucci E, Smith-Warner SA, et al. Intakes of fruits, vegetables, vitamins $A, C$, and E, and carotenoids and risk of renal cell cancer. Cancer Epidemiol Biomarkers Prev 2006; 15:2445-52.

73. Hu J, La Vecchia C, Negri E, et al. Dietary vitamin C, E, and carotenoid intake and risk of renal cell carcinoma. Cancer Causes Control 2009;20:1451-8.
74. Bertoia M, Albanes D, Mayne ST, et al. No association between fruit, vegetables, antioxidant nutrients and risk of renal cell carcinoma. Int J Cancer 2010;126:1504-12.

75. van Dijk BA, Schouten $\mathrm{L}$, Oosterwijk E, et al. Carotenoid and vitamin intake, von Hippel-Lindau gene mutations and sporadic renal cell carcinoma. Cancer Causes Control 2008;19:125-34.

Correspondence: Dr. Jehonathan H. Pinthus, Associate Professor, Department of Surgical Oncology, Juravinski Cancer Centre, 699 Concession Street, Hamilton, ON L8V 5C2; jehonathan.pinthus@jcc.hhsc.ca

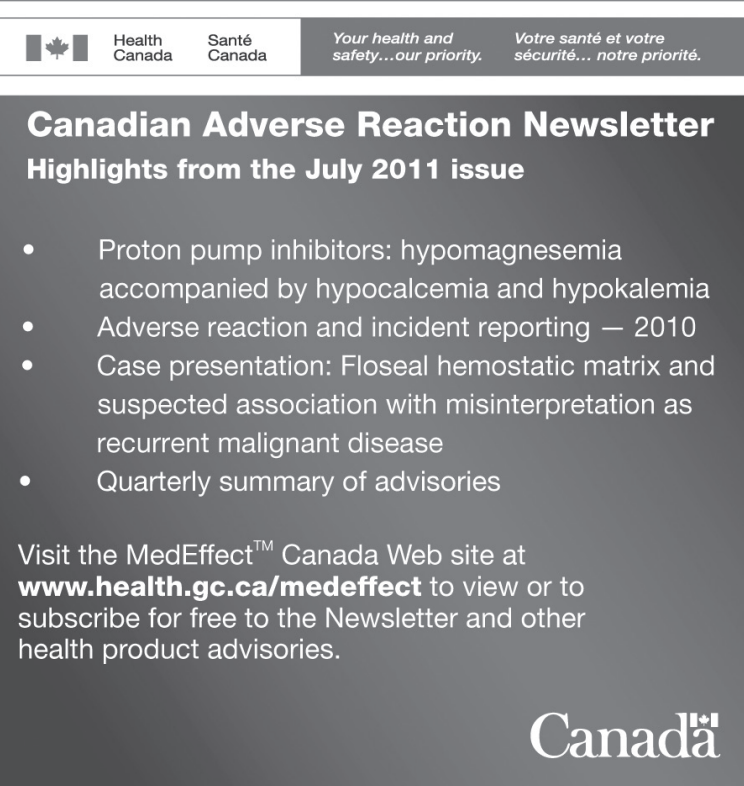

